\title{
Disease burden of post-infectious irritable bowel syndrome in The Netherlands
}

\author{
J. A. HAAGSMA ${ }^{1,2 *}$, P. D. SIERSEMA ${ }^{3}$, N. J. DE WIT ${ }^{4}$ AND A. H. HAVELAAR ${ }^{1,5}$ \\ ${ }^{1}$ Centre for Infectious Disease Control, National Institute for Public Health and the Environment, Bilthoven, \\ The Netherlands \\ ${ }^{2}$ Department of Public Health, Erasmus Medical Center, Erasmus University Rotterdam, Rotterdam, \\ The Netherlands \\ ${ }^{3}$ Department of Gastroenterology and Hepatology, University Medical Center Utrecht, Utrecht, The Netherlands \\ ${ }^{4}$ Julius Center for Health Sciences and Primary Care, University Medical Center Utrecht, Utrecht, \\ The Netherlands \\ ${ }^{5}$ Institute for Risk Assessment Sciences, Utrecht University, Utrecht, The Netherlands
}

(Accepted 11 February 2010; first published online 12 March 2010)

\section{SUMMARY}

Post-infectious irritable bowel syndrome (PI-IBS) has been established as a sequel of infectious intestinal disease (IID). The aim of this study was to estimate the burden of PI-IBS caused by the pathogens Campylobacter, Salmonella and Shigella, and to compare this with other outcomes associated with these pathogens. The attributable risk of PI-IBS due to bacterial pathogens was calculated and linked to national data on gastroenteritis incidence and measures for severity and duration of illness in order to estimate the burden of PI-IBS. One year post-infection, IBS developed in $9 \%$ of patients with bacterial IID. The burden of PI-IBS adds over 2300 disability adjusted life years to the total annual disease burden for the selected pathogens. PI-IBS is a frequent sequel of IID, resulting in a considerable disease burden compared to other outcomes. If this relationship is not considered, this will result in an underestimation of the disease burden of IID.

Key words: Campylobacter, foodborne infections, public health, salmonellosis, Shigella.

\section{INTRODUCTION}

Over the past decade, burden of disease estimates have become of increasing significance in allocating medical resources, in targeting interventions, and for monitoring possible effect. These studies can use monetary units to quantify the burden of disease, so-called cost-of-illness studies, or can assess the

* Author for correspondence: J. A. Haagsma, M.Sc., National Institute for Public Health and the Environment, Centre for Infectious Disease Control, Laboratory for Zoonoses and Environmental Microbiology, P.O. Box 1, 3720 BA Bilthoven, The Netherlands.

(Email: j.haagsma@erasmusmc.nl) disease burden in a population by utilizing the disability adjusted life years (DALYs) metric [1].

The DALY integrates the effect of mortality as well as disease and disability, which allows them to be considered at the same time. This simplifies comparisons between distinct disease outcomes and subgroups of a population. A prerequisite for these comparisons, either in monetary units or in DALYs, is that the burden of disease comprises a complete assessment of the health effect due to a certain pathogen. This means for infectious intestinal disease (IID) that, apart from the direct consequence of gastroenteritis, all sequelae should be considered. In previous studies 
Table 1. Population attributable risk of developing IBS following gastroenteritis, per study

\begin{tabular}{|c|c|c|c|c|c|}
\hline \multirow[b]{2}{*}{ Study } & \multirow[b]{2}{*}{ Aetiology } & \multirow{2}{*}{$\begin{array}{l}\text { Follow-up } \\
\text { (months) }\end{array}$} & \multicolumn{2}{|c|}{ Incidence of IBS } & \multirow[b]{2}{*}{$\operatorname{AR}(\%)$} \\
\hline & & & Cases $(\%)$ & Controls $(\%)$ & \\
\hline Rodriguez \& Ruigomez [26] & Bacterial & 12 & $14 / 318(4 \cdot 4)$ & $2027 / 584308(0 \cdot 3)$ & $4 \cdot 1$ \\
\hline Parry et al. [27] & $\begin{array}{l}\text { Campylobacter } \\
\text { Salmonella }\end{array}$ & 6 & $18 / 108(16 \cdot 7)$ & $4 / 206(1 \cdot 9)$ & $14 \cdot 8$ \\
\hline Wang et al. [37] & Shigella & 10 & $24 / 235(10 \cdot 2)$ & $2 / 243(0 \cdot 8)$ & $9 \cdot 4$ \\
\hline Mearin et al. [34] & Salmonella & 12 & $31 / 266(13 \cdot 2)$ & $5 / 333(1 \cdot 5)$ & $11 \cdot 7$ \\
\hline Jung et al. [14] & Shigella & 12 & $12 / 87(13 \cdot 8)$ & $1 / 88(1 \cdot 1)$ & $12 \cdot 7$ \\
\hline
\end{tabular}

IBS, Irritable bowel syndrome; AR, attributable risk.

on the burden of IID [2-4], we included outcomes, such as Guillain-Barré syndrome, reactive arthritis, inflammatory bowel disease and haemolytic uraemic syndrome, leading to chronic renal failure to complement the burden of acute gastroenteritis.

Previous studies have shown that the prevalence of irritable bowel syndrome (IBS) in Western populations is between $10 \%$ and $20 \%[5,6]$. There is growing evidence of persisting gastrointestinal symptoms after bacterial IID, and these patients will, at least partly, meet the diagnostic criteria for (postinfectious) irritable bowel syndrome (PI-IBS) [7-11]. Although not life-threatening or severely debilitating, IBS symptoms may have a severe effect on daily life, affecting work, school and social life, and decreasing health-related quality of life over long periods $[12,13]$. These periods of decreased health-related quality of life on the one hand and its high prevalence in society on the other might add up to a substantial burden of disease, which is currently not considered in estimates of the burden of IID.

Nonetheless, when PI-IBS is considered in such studies, the background IBS population prevalence and the percentage attributable to IID should be indisputably clear. For this purpose, the attributable risk (AR) has been established as the preferred measure of association.

In the current study, we first aimed to assess the AR of developing PI-IBS for bacterial pathogens. Second, we estimated the disease burden in DALYs of PI-IBS due to a particular group of pathogens, i.e. (thermophilic) Campylobacter spp., Salmonella spp., and Shigella spp. in The Netherlands. These pathogens were chosen because they are the most frequent causes of bacterial IID and have been demonstrated to be responsible for the largest burden of IID [2]. Third, we compared this burden with other outcomes associated with these pathogens.

\section{METHODS}

\section{Literature review}

We based our study on the recently published metaanalysis by Thabane et al. [11]. From this paper we selected four studies (Table 1) in which:

- IID patients were considered who had no previous history of IBS or other bowel disorders.

- Appropriate control groups were included.

- A bacterial aetiology was confirmed by stool culture from at least a proportion of the patients with IID.

In an additional search in the recent literature, we identified one study [14] that met the same selection criteria. This study presented follow-up data of a previously reported cohort $[15,16]$, and we therefore only used the most recent of these two studies in our evaluation. If available, we used data after a followup period of 1 year, or as close to this time point as possible. Studies involving patients with traveller's diarrhoea were excluded because it has been suggested that these are predominantly caused by enterotoxigenic E. coli, which has a milder course and a lower risk of developing PI-IBS [17].

\section{AR}

The AR was calculated as the additional incidence rate (IR) of exposed (IID) cases compared to unexposed (non-diseased) controls:

$A R=I R_{\text {exposed }}-I_{\text {unexposed }}$.

The uncertainty in the AR was modelled by employing a Bayesian approach [18]. Both $\mathrm{IR}_{\text {exposed }}$ and $\mathrm{IR}_{\text {unexposed }}$ cases were considered as binomial fractions in which the uncertainty was modelled as a $\operatorname{Beta}(s, f)$ distribution, in which $s$ is the number of 
persons in the exposed or unexposed group who developed IBS and $f$ is the number of persons who did not develop IBS. With this approach, the $95 \%$ confidence interval (CI) is similar to that estimated by frequentist statistics, with the advantage that now a full uncertainty distribution can be simulated.

\section{DALYs}

The incidence of gastrointestinal infectious disease due to Campylobacter, Salmonella and Shigella was derived from national incidence data on foodborne disease, based on a method reported by Havelaar et al. [19]. The estimates were updated to the year 2006 (for details, see [2]). These incidence data relate to all cases in the population and are based on data from Sensor, a Dutch community-based cohort study [20]. By combining the incidence of gastrointestinal infectious disease associated with the selected pathogens and a weighted mean AR, the incidence of PIIBS was calculated. The estimated incidence of PI-IBS was then used to calculate the burden of PI-IBS in DALYs. Uncertainty in incidence and DALY estimates was quantified by Monte Carlo simulation.

The DALY aggregates mortality, expressed in years of life lost (YLL) and morbidity, expressed in years lived with disability (YLD), according to the following calculation:

$D A L Y=Y L L+Y L D$,

where YLL represents the time lost due to premature mortality. For PI-IBS the mortality component of the DALY is not taken into account as IBS is not associated with increased mortality. YLD represents the time in good health lost while living with a disease or disability and is calculated with the following formula:

$\mathrm{YLD}=\Sigma n_{l} * t_{l} * d w_{l}$,

where $n_{l}$ is the number of cases with health outcome $l$, $t_{l}$ the duration of the health outcome and $d w_{l}$ the disability weight assigned to health outcome. The disability weight is a value ranging from 0 to 1 that is assigned to living with a medical condition. This value reflects the effect of a specific health condition on health-related quality of life and is commonly based on the preferences of an expert or lay panel. We adopted the IBS disability weight from the Mild Diseases and Ailments Study, a Dutch study that generated disability weights for 52 health conditions from panels of medical experts and laymen recruited from

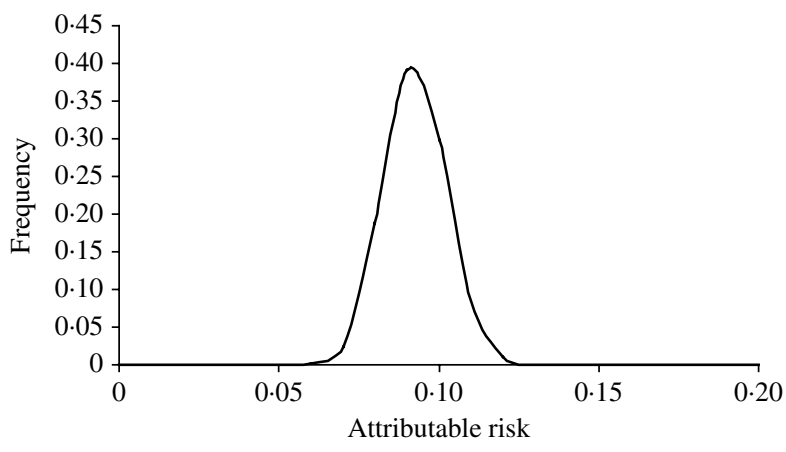

Fig. 1. Uncertainty distribution of the attributable risk of post-infectious irritable bowel syndrome in infectious intestinal disease patients with bacterial aetiology.

the general population. In this study a renewed methodology was applied that focused especially on obtaining and improving disability weights for functional losses of a temporary and complex nature [21]. This can also be applied to IBS symptoms, because they tend to subside and exacerbate with periods of partial remission. To align with the societal point of view of the DALY, we used the IBS disability weight derived from the population panel $(n=105)$ which had a value of $0 \cdot 042$. For the duration of PI-IBS, several long-term follow-up studies were assessed $[15,16,22-25]$. Based on these studies, we assumed for PI-IBS provoked by bacteria an average duration of 5 years [14].

\section{RESULTS}

Table 1 presents an overview of the results of the five case-control studies that analysed the incidence of PI-IBS following IID. In these studies, the index group was a cohort of gastroenteritis patients with confirmed bacterial aetiology. Controls in these studies were either volunteers, patients from the same general practice or siblings or spouses of the patient. The PI-IBS incidence rate in these five studies ranged from $4 \%$ [26] up to $17 \%$ [27]. The calculated AR is also shown in Table 1. These data do not suggest that PI-IBS is different for various bacterial pathogens ( $\chi^{2}$ test for homogeneity, $P=0.95$ [28]). We therefore pooled the studies considering patients with bacterial aetiology, weighted by the number of cases that experienced an episode of IID. This resulted in a mean weighted AR for bacterial infections (10-12 months post-IID) of $8 \cdot 8 \%(90 \%$ CI $7 \cdot 2-10 \cdot 4$; Fig. 1$)$.

In The Netherlands, about 124000 cases of IID due to the three pathogens were considered in 2006 
Table 2. Incidence of infectious intestinal disease due to three pathogens in The Netherlands (population 16.3 million), 2006

\begin{tabular}{llcc}
\hline \hline Pathogen & Total cases & GP visits & $\begin{array}{l}\text { Reported } \\
\text { cases }\end{array}$ \\
\hline Salmonella & $42000(8800-110000)^{*}$ & $7300(4300-11000)$ & 1700 \\
Campylobacter & $78000(28000-170000)$ & $19000(10000-3000)$ & 3400 \\
Shigella & $4400(600-11000)$ & $700(100-1800)$ & 240 \\
\hline \hline
\end{tabular}

GP, General Practitioner.

* Mean (5th-95th percentile).

Table 3. Incidence and prevalence of post-infectious irritable bowel syndrome due to three pathogens in The Netherlands, 2006

\begin{tabular}{lll}
\hline \hline Pathogen & Incidence & Prevalence* \\
\hline Salmonella & $3700(750-9300) \dagger$ & $19000(3800-46000)$ \\
Campylobacter & $6900(2400-15000)$ & $34000(12000-74000)$ \\
Shigella & $370(50-960)$ & $1800(260-4800)$ \\
\hline \hline
\end{tabular}

* Incidence $\times 5$ years.

$\dagger$ Mean (5th-95th percentile).

(Table 2). Most of these IID cases were caused by Campylobacter (78000), and Salmonella (43000). About $27000 \mathrm{GP}$ visits were registered due to these three pathogens.

The three pathogens were estimated to be associated with about 11000 new cases of PI-IBS per year (Table 3), in this way contributing 55000 cases to the overall prevalence of IBS in The Netherlands; which is estimated to be 330000 formally diagnosed patients [5].

The disease burden of PI-IBS is shown in Table 4. In 2006, an estimated 2302 DALYs were lost due to PI-IBS following IID induced by the three selected pathogens, with $63 \%$ of DALYs due to Campylobacter, $34 \%$ to Salmonella and $3 \%$ to Shigella. Including PI-IBS in this calculation increased the burden of disease (Fig. 2) for Salmonella by $86 \%$ (from 905 to 1686 DALYs), for Campylobacter by $92 \%$ (from 1564 to 3008 DALYs) and for Shigella by $151 \%$ (from 51 to 128 DALYs).

\section{DISCUSSION}

Based on recent findings in the literature, we estimated that about $9 \%$ of patients with bacterial IID will develop PI-IBS 1 year after infection [11]. We also estimated that about 55000 prevalent cases of PI-IBS in the Dutch population will actually result from an infection with Salmonella, Campylobacter or Shigella. This is about $17 \%$ of the total prevalence of formally diagnosed PI-IBS. Others [29] have estimated a similar range of $6-17 \%$.

Our study used data from case-control studies to assess the incidence and disease burden of PI-IBS. Over the past few years, the evidence that an episode of IID is a risk factor for developing IBS has increasingly been recognized, reinforced by a growing understanding of the pathophysiology of IBS [30-32]. In brief, mucosal injury and inflammation are assumed to increase the number of enterochromaffin cells, thus increasing the release of serotonin (5-hydroxytryptamine, 5-HT). 5-HT affects gastrointestinal motility, enterocyte secretion and visceral sensation. Increased levels of pro-inflammatory cytokines and lymphocyte counts in intestinal epithelium and lamina propria have indeed been documented in PI-IBS patients [7].

In a recent follow-up study, 3 years after a casecontrol study on laboratory-confirmed cases of Campylobacter and Salmonella, an increased risk of IBS could not be confirmed [33]. In contrast, the results of this study suggested that patients with IBS or with another chronic intestinal condition were more susceptible to IID. The studies identified in our literature review excluded cases with pre-existing symptoms; hence, we only included cases in which the IID episode triggered a new case of IBS. Patients with already existing IBS or another intestinal illness were not included in our study.

In the current study, only limited information was available on some other factors involved in PI-IBS, and this may have affected our results in the following way. First, the data used to estimate the incidence of PI-IBS were retrieved from the existing literature. Several case-control studies were used and the time interval chosen for follow-up of exposed 
Table 4. Disease burden of post-infectious irritable bowel syndrome due to three pathogens in The Netherlands, 2006

\begin{tabular}{llllr}
\hline \hline Pathogen & Incidence & $\begin{array}{l}\text { Duration } \\
\text { (years) }\end{array}$ & $\begin{array}{l}\text { Disability } \\
\text { weight }\end{array}$ & $\begin{array}{l}\text { Disease burden } \\
\text { (YLD) }\end{array}$ \\
\hline Salmonella & $3700(750-9300)^{*}$ & 5 & $0 \cdot 042$ & $780(160-2000)^{*}$ \\
Campylobacter & $6900(2400-15000)$ & 5 & $0 \cdot 042$ & $1400(510-3100)$ \\
Shigella & $370(50-960)$ & 5 & $0 \cdot 042$ & $80(10-200)$ \\
\hline \hline
\end{tabular}

YLD, Years lived with disability.

* Mean (5th-95th percentile).

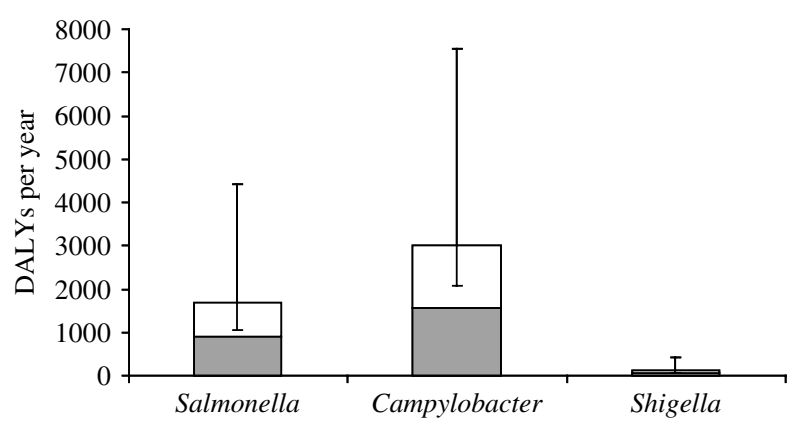

Fig. 2. Disease burden in disability adjusted life years (DALYs) per year due to three pathogens in The Netherlands, 2006, including ( $\square$ ) and excluding ( $\square$ ) postinfectious irritable bowel syndrome. Error bars express the 5th and 95th percentiles resulting from Monte Carlo simulation.

and non-exposed participants varied from 3 months [15] to 5 years [14]. Overall, we found that the incidence of PI-IBS in exposed participants was higher with a shorter follow-up period [22, 23, 34]. In order to present a pooled estimate we chose a follow-up period of 10-12 months. Using shorter follow-up periods would have resulted in an increased AR, and, subsequently, a higher estimated incidence of PI-IBS. As these additional cases had a disease duration of $<1$ year, they would have added little value to the total burden of PI-IBS.

Second, in this study we did not consider patient characteristics (such as gender, smoking or psychosocial comorbidity) as risk factors for developing PI-IBS. Similarly, we did not take into account the details of the gastroenteritis episode, such as duration or aetiology of the infection [10, 22, 27, 35, 36]. With regard to duration of gastroenteritis, previous studies revealed that the longer period patients have symptoms due to gastroenteritis or the larger effect these symptoms have, the higher the risk of having PI-IBS $[36,37]$. We were not able to adjust for IID duration or severity, due to a lack of comparison.
Third, the disability weight is based on the preferences of a population panel, which is in line with the societal perspective of the DALY metric. Nevertheless, the IBS disability weight is not able to capture the heterogeneous group of clinical symptoms that are experienced by patients. Several studies have shown that there is a wide variation in symptoms between patients [38]. As a result, actual health states of patients with (PI-)IBS may differ considerably from the health state descriptions valued by the population panel. Moreover, the value of the disability weight seems to be rather low. Health-related quality-of-life studies performed in patients with IBS have shown that the loss of health-related quality of life was indeed considerable [13, 39]. This is not reflected by the value of the disability weights as applied in this study.

A case-control study by Marshall et al. has suggested that PI-IBS may also result from presumed viral IID [23]. As many as $20 \%$ (95\% CI 11-29) of PI-IBS cases developed in IID patients, although this risk was only statistically significant after 3 months. This shorter duration of PI-IBS due to viral IID is in line with the observations of Spiller \& Garsed [29], who noted that recent studies have demonstrated that there is very little mucosal destruction in viral IID and, therefore, that the mucosal structure is likely to rapidly return to normal. When the shorter duration is taken into account, PI-IBS due to norovirus would still accumulate to a prevalence estimate of 32000 cases in the population and a disease burden of $1300 \mathrm{YLD}$, which is comparable to the disease burden of PI-IBS due to Campylobacter infection. This implies that including PI-IBS in disease burden estimates of the selected bacterial pathogens might still underestimate the burden of disease of foodborne disease. Further follow-up studies are needed that provide more information on the risk of IBS after viral IID. 
The results from the current study show that PI-IBS is a frequent sequel of IID and that the disease burden of PI-IBS is considerable compared to other outcomes of IID. The resulting annual non-fatal burden of disease of PI-IBS is over 2300 YLD, almost doubling the total non-fatal burden of disease for the selected pathogens. Ignoring PI-IBS in burdenof-disease studies results in an underestimation of the size of the burden of these types of gastrointestinal diseases. In order to satisfy the aspirations of burdenof-disease studies, i.e. identifying priorities in medical resource allocation, targeting of interventions and monitoring possible effect, all sequelae of IID should be included, given that the evidence on the association is sufficiently conclusive. There is definitely more research required on the duration of PI-IBS and related fluctuations in health-related quality of life, as well as on the association of viral gastroenteritis and PI-IBS.

\section{ACKNOWLEDGEMENTS}

The authors thank Yvonne van Duynhoven, Wilfrid van Pelt, Marion Koopmans, Erwin Duizer (National Institute for Public Health and the Environment, Bilthoven, The Netherlands), Claudia Stein (World Health Organization, Geneva, Switzerland), Martyn Kirk (Australian National University, Canberra, Australia) and Fred Angulo (Centers for Disease Control and Prevention, Atlanta, GA) for stimulating discussions and feedback on the draft manuscript.

\section{DECLARATION OF INTEREST}

None.

\section{REFERENCES}

1. Murray CJL, Lopez AD (eds). The global Burden of Disease: A Comprehensive Assessment of Mortality and Disability from Diseases, Injuries, and Risk Factors in 1990 and Projected to 2020. Cambridge, MA: Harvard University Press, 1996.

2. Kemmeren JM, et al. Priority setting of foodborne pathogens. Disease burden and costs of selected enteric pathogens. Bilthoven, The Netherlands: National Institute of Public Health and the Environment, 2006.

3. Cressey P, Lake R. Risk ranking: estimates of the burden of foodborne disease for New Zealand. Christchurch, New Zealand: ESR, 2007.

4. Havelaar AH, et al. Health burden in the Netherlands due to infection with thermophilic Campylobacter spp. Epidemiology and Infection 2000; 125: 505-522.
5. Hungin AP, et al. The prevalence, patterns and impact of irritable bowel syndrome: an international survey of 40,000 subjects. Alimentary Pharmacology \& Therapeutics 2003 ; 17 : 643-650.

6. Mertz HR. Irritable bowel syndrome. New England Journal of Medicine 2003; 349: 2136-2146.

7. DuPont AW. Postinfectious irritable bowel syndrome. Clinical Infectious Diseases 2008; 46: 594-599.

8. Halvorson HA, Schlett CD, Riddle MS. Postinfectious irritable bowel syndrome - a meta-analysis. American Journal of Gastroenterology 2006; 101 : 1894-1899.

9. Smith JL, Bayles D. Postinfectious irritable bowel syndrome: a long-term consequence of bacterial gastroenteritis. Journal of Food Protection 2007; 70: 17621769.

10. Spiller RC. Role of infection in irritable bowel syndrome. Journal of Gastroenterology 2007; 42: S41S47.

11. Thabane M, Kottachchi DT, Marshall JK. Systematic review and meta-analysis: the incidence and prognosis of post-infectious irritable bowel syndrome. Alimentary Pharmacology \& Therapeutics 2007; 26: 535-544.

12. Glia A, Lindberg G. Quality of life in patients with different types of functional constipation. Scandinavian Journal of Gastroenterology 1997; 32: 1083-1089.

13. Pare $\mathbf{P}$, et al. Health-related quality of life, work productivity, and health care resource utilization of subjects with irritable bowel syndrome: baseline results from LOGIC (Longitudinal Outcomes Study of Gastrointestinal Symptoms in Canada), a naturalistic study. Clinical Therapeutics 2006; 28: 1726-1735.

14. Jung IS, et al. The clinical course of postinfectious irritable bowel syndrome: a five-year follow-up study. Journal of Clinical Gastroenterology 2009; 43: 534-540.

15. Ji S, et al. Post-infectious irritable bowel syndrome in patients with Shigella infection. Journal of Gastroenterology and Hepatology 2005; 20 : 381-386.

16. Kim HS, et al. The development of irritable bowel syndrome after Shigella infection: 3 year follow-up study. Korean Journal of Gastroenterology 2006; 47 : 300 305.

17. DuPont HL. Systematic review: prevention of travellers' diarrhoea. Alimentary Pharmacology \& Therapeutics 2008; 27 : 741-751.

18. Vose D. Risk Analysis: A Quantative Guide. Chichester: John Wiley and Sons, 2000.

19. Havelaar AH, et al. Disease burden in The Netherlands due to infections with Shiga toxin-producing Escherichia coli O157. Epidemiology and Infection 2004; 132: 467-484.

20. de Wit MA, et al. Sensor, a population-based cohort study on gastroenteritis in the Netherlands: incidence and etiology. American Journal of Epidemiology 2001; 154: 666-674.

21. Janssen MF, Birnie E, Bonsel G. Feasibility and reliability of the annual profile method for deriving QALYs for short-term health conditions. Medical Decision Making 2008; 28: 500-510. 
22. Gwee KA, et al. Psychometric scores and persistence of irritable bowel after infectious diarrhoea. Lancet 1996; 347: 150-153.

23. Marshall JK, et al. Postinfectious irritable bowel syndrome after a food-borne outbreak of acute gastroenteritis attributed to a viral pathogen. Clinical Gastroenterology and Hepatology 2007; 5: 457-460.

24. McKeown ES, et al. Postinfectious irritable bowel syndrome may occur after non-gastrointestinal and intestinal infection. Neurogastroenterology and Motility 2006; 18: 839-843.

25. Neal KR, Barker L, Spiller RC. Prognosis in postinfective irritable bowel syndrome: a six year follow up study. Gut 2002; 51: 410-413.

26. Rodriguez LA, Ruigomez A. Increased risk of irritable bowel syndrome after bacterial gastroenteritis: cohort study. British Medical Journal 1999; 318: 565-566.

27. Parry SD, et al. Does bacterial gastroenteritis predispose people to functional gastrointestinal disorders? A prospective, community-based, case-control study. American Journal of Gastroenterology 2003 ; 98: 1970-1975.

28. Fisher LD, van Belle G. Biostatistics: A Methodology for the Health Sciences. New York: John Wiley and Sons, 1993.

29. Spiller R, Garsed K. Postinfectious irritable bowel syndrome. Gastroenterology 2009; 136: 1979-1988.

30. Crowell MD, et al. Enterochromaffin cells and 5-HT signaling in the pathophysiology of disorders of gastrointestinal function. Current Opinion in Investigational Drugs 2004; 5: 55-60.

31. Dunlop SP, et al. Abnormalities of 5-hydroxytryptamine metabolism in irritable bowel syndrome. Clinical Gastroenterology and Hepatology 2005; 3: 349-357.
32. Houghton LA, Foster JM, Whorwell PJ. Alosetron, a 5-HT3 receptor antagonist, delays colonic transit in patients with irritable bowel syndrome and healthy volunteers. Alimentary Pharmacology \& Therapeutics $2000 ; 14$ : 775-782.

33. Doorduyn Y, et al. Novel insight in the association between salmonellosis or campylobacteriosis and chronic illness, and the role of host genetics in susceptibility to these diseases. Epidemiology and Infection 2008; 136: 1225-1234.

34. Mearin F, et al. Dyspepsia and irritable bowel syndrome after a Salmonella gastroenteritis outbreak: oneyear follow-up cohort study. Gastroenterology 2005; 129: $98-104$.

35. Neal KR, Hebden J, Spiller R. Prevalence of gastrointestinal symptoms six months after bacterial gastroenteritis and risk factors for development of the irritable bowel syndrome: postal survey of patients. British Medical Journal 1997; 314: 779-782.

36. Cuomo R, Savarese MF, Gargano R. Almost all irritable bowel syndromes are post-infectious and respond to probiotics: consensus issues. Digestive Diseases 2007; 25: 241-244.

37. Wang LH, Fang XC, Pan GZ. Bacillary dysentery as a causative factor of irritable bowel syndrome and its pathogenesis. Gut 2004; 53: 1096-1101.

38. Bijkerk CJ, et al. Randomized patients in IBS research had different disease characteristics compared to eligible and recruited patients. Journal of Clinical Epidemiology 2008; 61: 1176-1181.

39. Brazier J, et al. A comparison of the EQ-5D and SF-6D across seven patient groups. Health Economics 2004; 13: $873-884$. 